\title{
Design and performance of the South Pole Acoustic Test Setup
}

\author{
Y. Abdou ${ }^{\mathrm{a}}$, K-H. Becker ${ }^{\mathrm{b}}$, J. Berdermann ${ }^{\mathrm{c}}$, M. Bissok ${ }^{\mathrm{d}}$, C. Bohm ${ }^{\mathrm{e}}$, S. Böser ${ }^{1 \mathrm{c}}$,

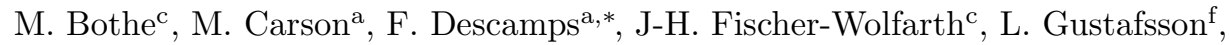

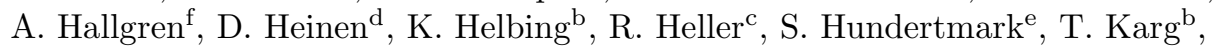 \\ K. Krieger ${ }^{c}$, K. Laihem ${ }^{\mathrm{d}}$, T. Meures ${ }^{\mathrm{d}}$, R. Nahnhauer ${ }^{\mathrm{c}}$, U. Naumann $^{\mathrm{b}}$, F. Oberson ${ }^{\mathrm{g}}$,

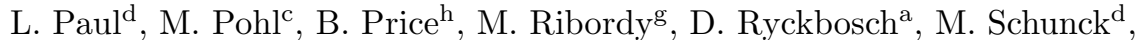

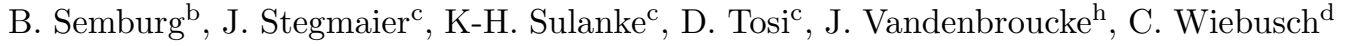 \\ ${ }^{a}$ Dept. of Subatomic and Radiation Physics, University of Gent, B-9000 Gent, Belgium \\ ${ }^{b}$ Dept. of Physics, University of Wuppertal, D-42119 Wuppertal, Germany \\ ${ }^{c}$ DESY, D-15735 Zeuthen, Germany \\ ${ }^{d}$ III. Physikalisches Institut, RWTH Aachen University, D-52056 Aachen, Germany \\ ${ }^{e}$ Oskar Klein Centre and Dept. of Physics, Stockholm University, SE-10691 Stockholm, Sweden \\ ${ }^{f}$ Dept. of Physics and Astronomy, Uppsala University, Box 516, S-75120 Uppsala, Sweden \\ ${ }^{g}$ Laboratory for High Energy Physics, École Polytechnique Fédérale, CH-1015 Lausanne, Switzerland \\ ${ }^{h}$ Dept. of Physics, University of California, Berkeley, CA 94720, USA
}

\begin{abstract}
The South Pole Acoustic Test Setup (SPATS) was built to evaluate the acoustic characteristics of the South Pole ice in the $10 \mathrm{kHz}$ to $100 \mathrm{kHz}$ frequency range, for the purpose of assessing the feasibility of an acoustic neutrino detection array at the South Pole. The SPATS hardware consists of four vertical strings deployed in the upper $500 \mathrm{~m}$ of the South Pole ice cap. The strings form a trapezoidal array with a maximum baseline of $543 \mathrm{~m}$. Each string has 7 stages equipped with one transmitter and one sensor module. Sound is detected or generated by piezoelectric ceramic elements inside the modules. Analogue signals are sent to the surface on electric cables where they are digitized by a PC-based data acquisition system. The data from all strings are collected on a central computer in the IceCube Laboratory from where they are send to a central data storage facility via a satellite link or stored locally on tape. A technical overview of SPATS and its performance is presented.
\end{abstract}

Keywords: SPATS, acoustic neutrino detection, glaciophone

PACS: 95.55.Vj, 43.58.-e, 43.58.Vb

\footnotetext{
${ }^{1}$ Now affiliated with the University of Bonn. Preprint submitted to Elsevier
} 


\section{Motivation and system requirements}

The sensitive volume needed for the detection of the predicted small cosmogenic neutrino flux and the study of its angular distribution is orders of magnitude larger than the instrumented volumes of the current Čerenkov neutrino telescopes 1, 2]. New detection methods that are sensitive to the radio or acoustic signatures of a UHE neutrino interaction would allow a more sparse instrumentation and therefore a larger sensitive volume at reasonable cost [3 $[6]$. The feasibility and specific design of an acoustic array as part of a hybrid optical/radio/acoustic neutrino detector, first suggested and simulated in [7], depends upon the acoustic properties of the South Pole ice in the concerned frequency region (10-100) kHz. The South Pole Acoustic Test Setup (SPATS) was built to evaluate the attenuation length, speed of sound, background noise level and transient noise rate of the South Pole ice and was deployed in the 2006/2007 and 2007/2008 polar seasons.

\section{Geometry}

The permanently installed hardware of SPATS consists of four instrumented cables, called strings, that were deployed vertically in the upper $500 \mathrm{~m}$ of selected IceCube [1] boreholes to form a trapezoidal array. The current geometrical configuration (see Fig. 1] and Fig. 2) is the result of a compromise between the geometry necessary to achieve the physics goals and the availability of IceCube holes and personnel availability during deployment.

Measuring, for example, the acoustic properties of the ice both parallel and perpendicular to the flow of the glacier permits the investigation of possible anisotropies of the acoustic properties of the ice. Therefore good horizontal coverage is needed. For an attenuation length analysis, it is important to have sufficient variation in transmitter to sensor distances so that the fit of amplitude versus distance is well constrained. The uncertainty on the horizontal position of each string is fixed and known to be $\pm 0.5 \mathrm{~m}$, so that the relative error decreases with increasing string-to-string distance (so called baseline). The vertical distance between the acoustic transducers was chosen to increase

\footnotetext{
* Corresponding author

Email address: Freija.Descamps@icecube.wisc.edu (F. Descamps)
} 


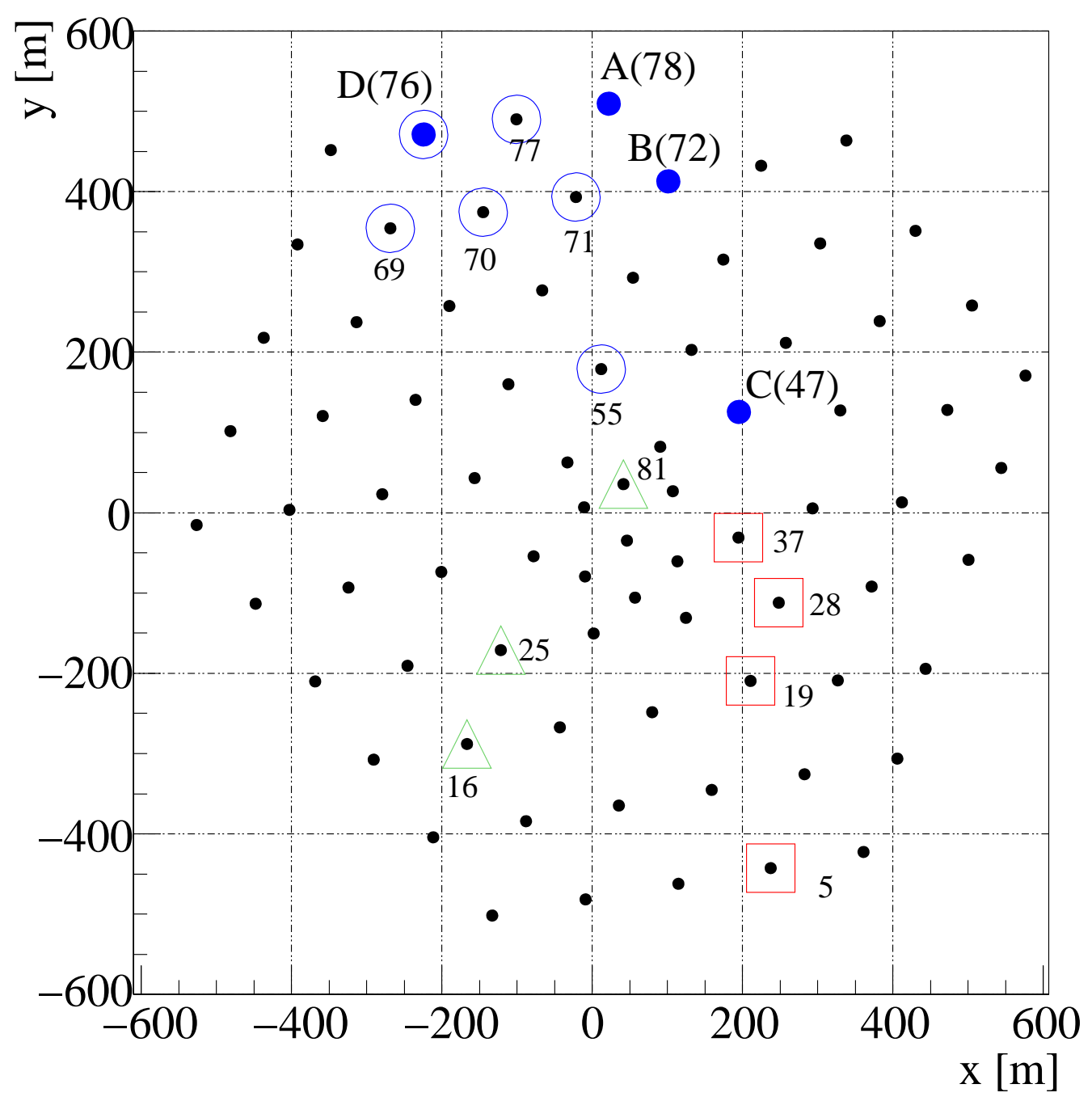

Figure 1: SPATS strings (large dots) overlayed on the IceCube geometry as of February 2009 (small dots). The String ID (A,B,C or D) is given followed by its corresponding IceCube hole number. The open circles, open squares and open triangles show the positions of the 2007/2008, 2008/2009 and 2009/2010 pinger holes (the pinger is a retrievable acoustic transmitter, see \$3.5) with the corresponding IceCube hole number. 


\begin{tabular}{c|c|c|c}
\hline \hline String & Deployed & Baseline $(\mathrm{m})$ & Instrumented depths $(\mathrm{m})$ \\
\hline \hline A & $14^{\text {th }}$ January 07 & (A-B) 125 & $80,100,140,190,250,320,400$ \\
B & $11^{\text {th }}$ January 07 & (B-C) 302 & $80,100,140,190,250,320,400$ \\
C & $22^{\text {nd }}$ December 06 & (C-A) 421 & $80,100,140,190,250,320,400$ \\
D & $24^{\text {th }}$ December 07 & (D-C) 543 & $140,190(\mathrm{H}), 250,320,400,430(\mathrm{H}), 500$ \\
\hline
\end{tabular}

Table 1: Deployment details for SPATS, the two HADES levels on String D are indicated.

with depth following the measured and predicted temperature and density profiles of the ice [8, 9]. Table 1 gives the deployment details and baselines for SPATS.

Figure 2 shows a schematic of the SPATS array with its in-ice and on-ice components. Each of the four strings has seven acoustic stages at specified depths. Table 1 shows the corresponding levels for each string. It was decided to leave out the two most shallow instrumented levels on String D after the 2007 data-set hinted towards less optimal acoustic conditions in those upper levels (higher background noise and shorter attenuation length). String D has second-generation SPATS transmitters and sensors. In addition, the Hydrophone for Acoustic Detection at South Pole (HADES, see [10]) was deployed on two levels of String D (190m and $430 \mathrm{~m})$. The acoustic modules are discussed in more detail in the following sections.

\section{In-ice components}

Each acoustic stage consists of a separate transmitter and sensor module. All the electronic circuits are located in stee 2 pressure housings which have an outer diameter of $101.6 \mathrm{~mm}$ and a wall thickness of $5.74 \mathrm{~mm}$. The transmitter and sensor modules are joined together with three ropes, allowing the transmitter module to be mounted about $45 \mathrm{~cm}$ above the sensor module (see Fig. 3). The ropes are attached at the top and bottom to a steel nut that is threaded and mounted on a bolt. This bolt also runs through a plastic perforated hollow sphere. The holes allow for water to flow in so that the spheres remain intact at least until freeze-in. These spacer balls are $16 \mathrm{~cm}$ in diameter and assure

\footnotetext{
${ }^{2}$ Stainless steel grade 304/1.4301
} 


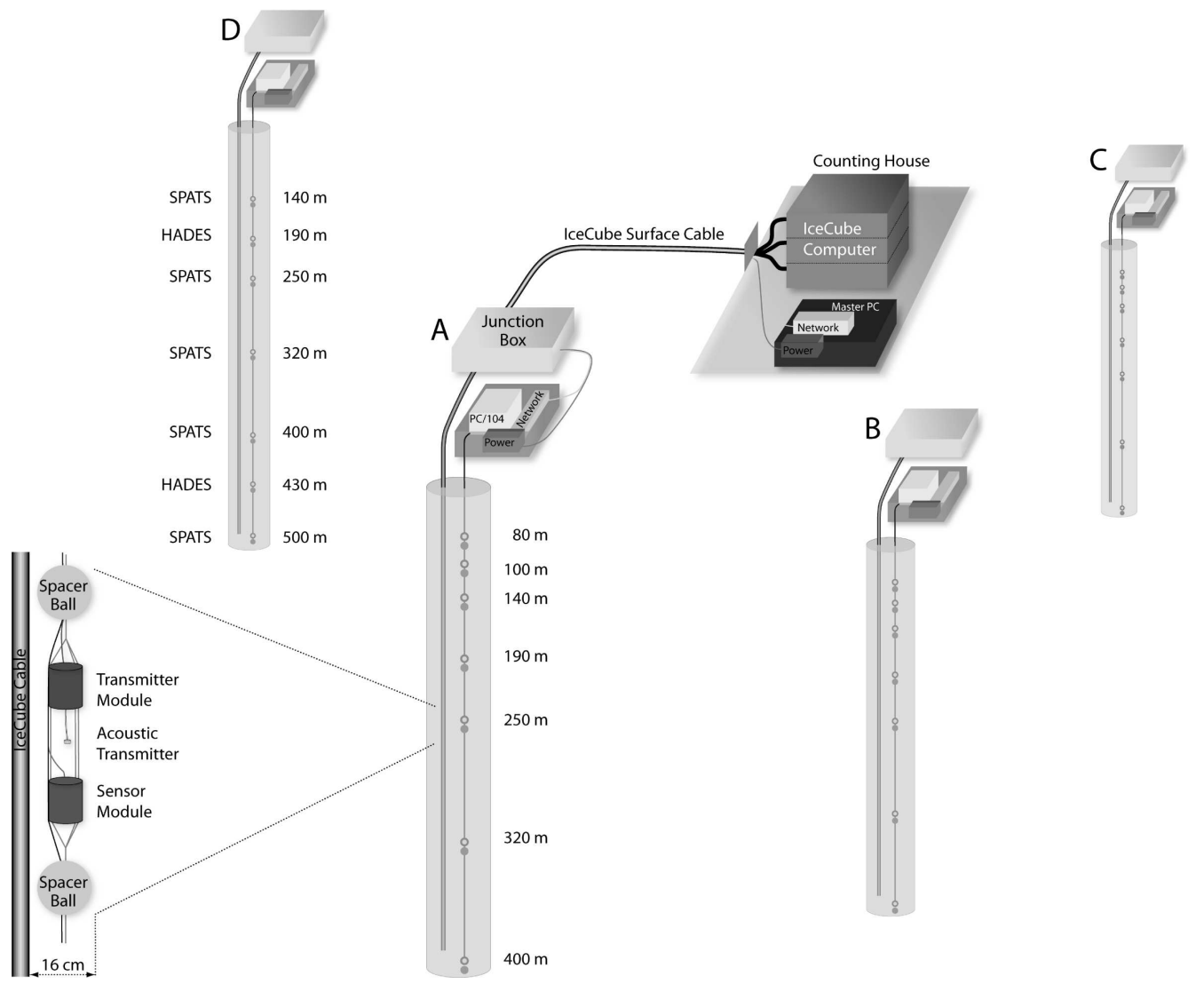

Figure 2: Schematic of the SPATS array, with the four strings consisting of seven acoustic stages.

a minimum distance of the stage to the IceCube main cable and the wall of the hole. An entire stage is about $1.5 \mathrm{~m}$ long and maximum $16 \mathrm{~cm}$ wide with a total weight of $10 \mathrm{~kg}$.

Each acoustic stage is connected to an Acoustic Junction Box (AJB), located at the surface, by two shielded cables (one for each module) each consisting of four twisted pairs. All in-ice cables are threaded around a support rope which carries the weight of the stages. The depth of the string was monitored during deployment by reading out the pressure sensor that is inside each of the bottom-most transmitter modules. The resulting precision in the depth measurement is estimated to be $\pm 2 \mathrm{~m}$.

\subsection{The transmitter module}

The SPATS transducers acoustic elements all consist of the same lead zirconium titanate (PZT) material, namely PIC151, manufactured by PI-Ceramics. This is a soft 


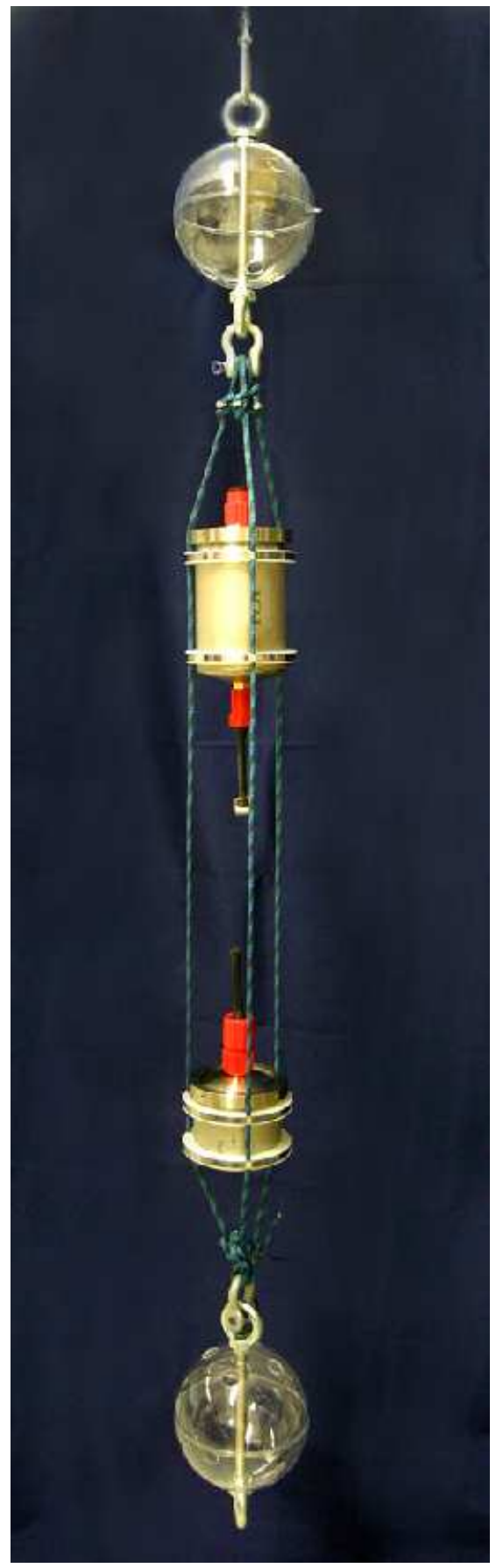

Figure 3: Fully assembled SPATS stage. 


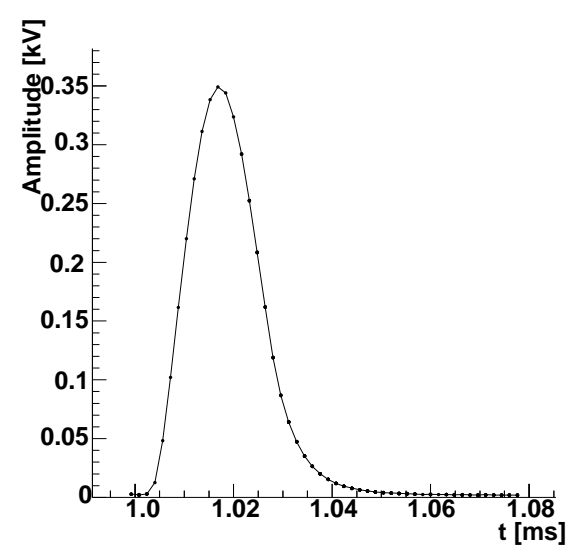

(a)

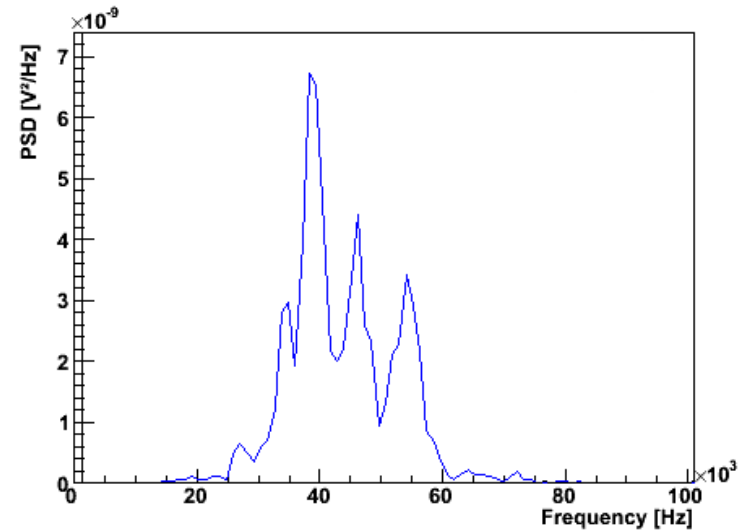

(b)

Figure 4: (a): An averaged high voltage pulse for the first generation SPATS transmitter. The statistical error bars are too small to be seen on this figure. (b): Fourier spectrum for a String B transmitter as seen by the HADES sensor at $430 \mathrm{~m}$ depth.

piezo-ceramic material with a high piezoelectric charge constant $\left(\mathrm{d}_{33}=500 \frac{p C}{N}\right)$, high permittivity and a high coupling factor.

The transmitter module consists of a steel pressure vessel that houses a high-voltage pulse generator board (hereafter HV-board) and a temperature sensor. For the deepest stages, the temperature sensor is replaced with a commercial pressure sensor. Both sensors give a linear output current range of (4-20) $\mathrm{mA}$, which translates to a temperature range of $-70^{\circ} \mathrm{C}$ to $+10^{\circ} \mathrm{C}$ and a pressure range of $(0-60)$ bar. The HV-board hosts a a circuit designed to pulse the piezoelectric ceramic with a high voltage pulse. An LC circuit is used as an intermediate stage to locally store the charge necessary for the generation of the HV pulse. The total charge accumulated is determined by two remotely controllable parameters: the length of the TTL trigger signal during which the LC circuit is charged and the DC steering voltage. When the TTL pulse goes to a high state, a current (defined by the DC steering voltage), flows across the LC stage. When the TTL pulse goes to a low state, the charge accumulated is discharged onto the piezo and an acoustic pulse is emitted. The charging voltage is variable and is provided by a voltage regulation board.

Gaussian shaped HV pulses with a FWHM of about $17 \mu$ s and a variable maximum up to $1.5 \mathrm{kV}$ are sent to the piezo-ceramic element. The resulting $\mathrm{HV}$ pulse is read 
back as a differential signal (HVRB). This allows effects of cable length and temperature to be studied and taken into account in subsequent analyses. Figure 4(a) shows an averaged HV pulse, averaged over ten individual pulses, generated with a first-generation SPATS transmitter module. This shows a very high repetition stability. These pulses are triggered by TTL signals of $5 \mathrm{~ms}$ duration. Figure 4(b) shows the Fourier spectrum for a waveform from HADES (see §3.3) listening to a String B transmitter (BT4). The SPATS transmitters typically output most power in the $20 \mathrm{kHz}$ to $60 \mathrm{kHz}$ range, although the variations of the actual shape of the emission spectra are large. The String D transmitters have an optimized HV-circuit design which results in sinusoidal half-wave HV pulses with a FWHM of about $50 \mu \mathrm{s}$ and higher pulse amplitudes. These pulses are triggered by TTL signals of typically $2 \mathrm{~ms}$ duration.

The ring-shaped piezo-ceramic element is cast in epoxy for electrical insulation and positioned $\sim 13 \mathrm{~cm}$ below the steel housing. Azimuthally isotropic emission is the motivation for the use of ring shaped piezo-ceramics. The actual emission directivity of such an element was measured in azimuthal and polar directions, see $\S 7.3$, The ring-shaped ceramic element is not isotropic in the polar plane. The HV cable that connects this element to the transmitter module has limited flexibility and an extra rigidity was added before deployment to avoid bending. Still, it can be expected that the piezo has a certain angle to the horizontal plane. In this case the amplitude variation in the horizontal plane will be larger than expected from a perfectly horizontally mounted piezo.

\subsection{The sensor module}

The sensor module has three channels placed $120^{\circ}$ apart in order to ensure good angular coverage. A channel consists of a cylindrical ( $10 \mathrm{~mm}$ diameter and $5 \mathrm{~mm}$ height) piezo-ceramic element of the PZT type that is pressed against the steel housing. The ceramic element is directly soldered to a 3 -stage amplifier, the first stage of which consists of a low-noise amplifiet 3 with an amplification factor of 100, a large bandwidth and a $3 \mathrm{kHz}$ high-pass filter to suppress low frequencies. The second amplifier stage is an AC coupled inverted amplifier that amplifies the signal by a factor of 100 and suppresses frequencies above $100 \mathrm{kHz}$. The last stage is a line driver: it prepares the signal for

\footnotetext{
${ }^{3} \mathrm{AD} 754 \mathrm{JR}$
} 
transport over the long in-ice cable by providing a differential output with a gain of 1 . Two twisted cables then transport the complementary signals to the differential input of the ADC in the string-PC. This reduces noise by rejecting common-mode interference. The total amplification factor of the chain of amplifiers is of the order of $10^{4}$.

The 21 first-generation sensor modules that were deployed on strings A, B and C have a central bolt that is connected to three screws. These preload screws put pressure on the ceramic element through the amplifier board to ensure good contact with the steel housing and avoid deformation of the module. This introduces a mechanical coupling between the three different channels of the sensor module. It was therefore decided to replace the preload screws with a metal ring in the second-generation sensor modules on String D. Figure 5 shows pictures of open first $5(\mathrm{a})$ and second $5(\mathrm{~b})$ generation

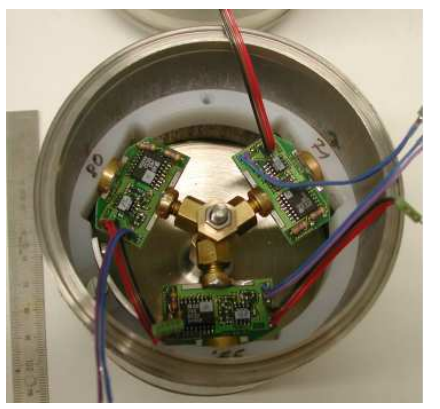

(a) SPATS first generation sensor module.

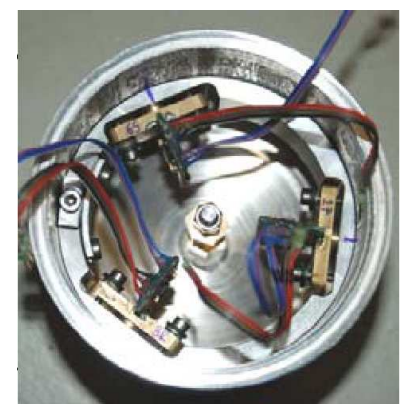

(b) SPATS second generation sensor module.

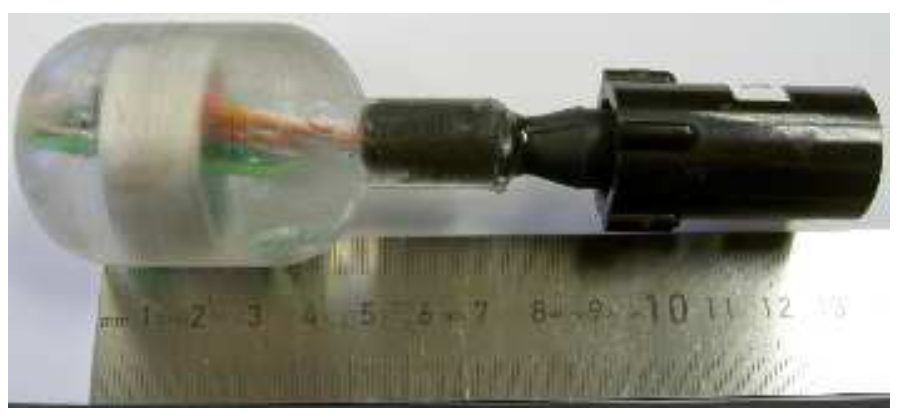

(c) HADES sensor.

Figure 5: Pictures of the SPATS and HADES sensors. The SPATS first (a) and second (b) generation sensor modules have 3 channels consisting of a piezo-ceramic element followed by an amplifier board. The HADES sensor (c) consists of a cylindrical piezo-ceramic element with a amplifier board cast in resin. 
SPATS sensor modules.

\section{3. $H A D E S$}

The Hydrophone for Acoustic Detection at South Pole (HADES) was developed in order to offer an alternative in-ice sensor with a different dynamical range. HADES-A and HADES-B were deployed on String D at depths of $190 \mathrm{~m}$ and $430 \mathrm{~m}$. A ring-shaped piezoceramic element is connected to a two-stage differential amplifier that is placed inside the ring. The assembly is then coated with resin (two component hard polyurethane). The choice of resin was made after a series of laboratory tests that investigated the resistance of the material to temperatures down to $-85^{\circ} \mathrm{C}$. Also, the acoustic impedance matching was calculated from the measured sound speed for each material. Figure $5(\mathrm{c})$ shows a picture of the HADES sensor.

\subsection{Naming conventions}

The SPATS strings were named String A, B, C and D. The three sensor channels of each sensor module are each read out by a different fADC board. Therefore the sensor channels are named channel 0,1 or 2 , based on the fADC board to which they are connected. A sensor channel is named $X \mathrm{~S} Y$-Z, pointing to the sensor channel $Z$ of the sensor module at position $Y$ on String $X$. The naming convention for a transmitter is $X$ T $Y$ : the transmitter on String $X$ at position $Y$. Stage positions $Y$ are number 1 to 7 from the top of the string to the bottom. Channel 0 of the top sensor of String A is designated as AS1-0.

\subsection{Pinger}

In addition to the permanently installed instrumentation which has been described above, a retrievable transmitter, called pinger, was used at the South Pole in the 2007/2008, $2008 / 2009$ and 2009/2010 seasons. A description of the pinger can be found in [11] and [12].

\section{On-ice components}

\subsection{The acoustic junction box}

The AJB is a robust aluminium box (dimension: $(30 \times 50 \times 80) \mathrm{cm}$ for strings $\mathrm{A}, \mathrm{B}$ and $\mathrm{C}$ and slightly larger for String D) that is situated above each acoustic string and buried 


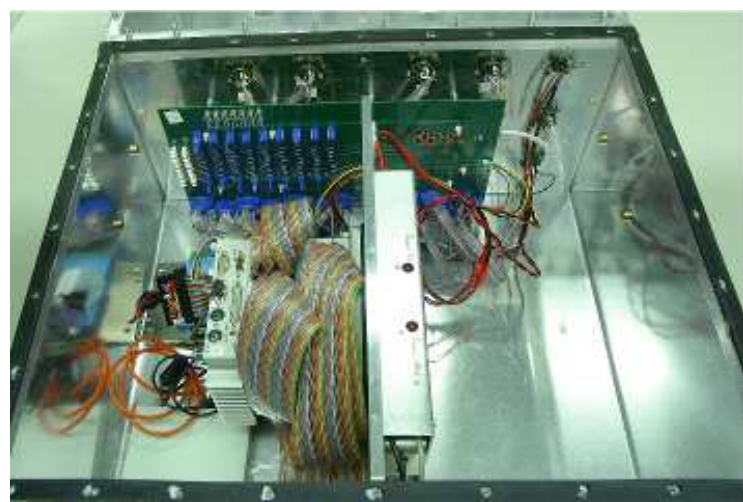

(a) A view of the AJB

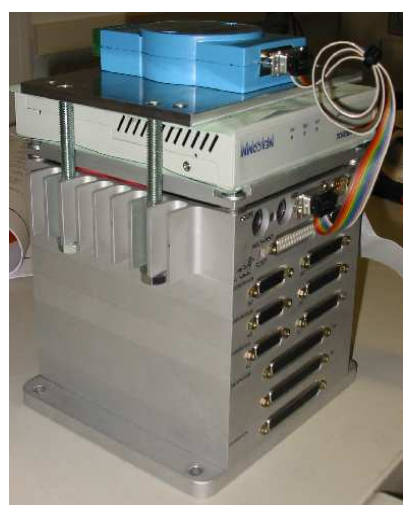

(b) The string-PC with DSL and serial modem.

Figure 6: (a): The watertight part of the acoustic junction box (AJB) with the PCB, power distribution board, string-PC and modems. (b): The string-PC stack. From top to bottom are the serial and DSL modems, the CPU board, 3 fast-ADC boards, 1 slow-ADC board and 1 relay board.

under roughly $3 \mathrm{~m}$ of snow. It is split into two compartments; one holds the connectors from the in-ice cables. These are connected to wall-mounted connector-sockets (patch wall). The other compartment is sealed watertight and contains the electronic components (see Fig. 6(a)) . All in-ice signals are first routed through a printed circuit board (PCB) from where they are distributed to the DAQ-boards of a low-power industrial $\mathrm{PC}$, called the string-PC. A power distribution unit (PDU) consists of low-noise DC/DC converters and filters. It provides ground, $+5 \mathrm{~V},+15 \mathrm{~V}$ and $+24 \mathrm{~V}$ to the string-PC and the in-ice components through the PCB. The PCB also routes the GPS timing signal and converts the currents from the in-ice pressure and temperature sensors to voltages.

\subsection{The string- $P C$}

The string-PC is a stack of IDAN (Intelligent Data Acquisition Node) PC/104 modules by RTD4 (see Fig. 6(b)). It has a compact modular design and its splash-proof rugged aluminium enclosure acts as a heat sink so that no fan is required. It is rated to perform from $-40^{\circ} \mathrm{C}$ to $+85^{\circ} \mathrm{C}$. The peripheral components are controlled by a CPU module 5 with a $600 \mathrm{MHz}$ processor and $512 \mathrm{MB}$ RAM. The Linux operating system is

\footnotetext{
${ }^{4}$ http://www.rtd.com/

${ }^{5}$ IDAN-CML47786HX650ER-260D
} 
installed on a flash-memory (wide temperature disk-on-module (DOM)6 $)$. This type of solid-state memory has the advantage that there are no moving parts, unlike a regular hard drive. The drawback is that each memory-cell of the DOM can be accessed for writing a limited number of times. If too many cells fail the disk will become unaccessible. For this reason, all runtime data are stored locally on a temporary RAM-disk. This means that part of the memory of the CPU module is treated as if it were a disk drive. Hence, the read-write cycles to the DOM are kept to a minimum. The 12-bit, 8 channel fADC board 7 have a maximum sampling frequency of $1.25 \mathrm{MHz}$. There are three fADCs for each string, which means that all three channels of one sensor module can be read out simultaneously at a maximum sampling frequency of $1.25 \mathrm{MHz}$. Theoretically, the fADCs should be able to read out all 21 sensor-channels of the string at maximum $\sim 179 \mathrm{kHz}$ sampling frequency. The three boards are linked through a SyncBus (RTD) connection which allows the simultaneous recording of samples (using the same ADC clock of one of the boards). Together with one slow ADC/DAC board8 with a sampling frequency of $500 \mathrm{kHz}$, the transmitters can be controlled and the temperature and pressure sensors can be read out.

A relay board 9 has 16 relays which allows the power for each sensor, transmitter and the power to the temperature and pressure sensors to be switched on and off individually. The average power consumption per string is low and varies from $\sim 35 \mathrm{~W}$ (no in-ice modules powered on) to $\sim 96 \mathrm{~W}$ (all in-ice modules powered-on). All these components were tested at the expected low temperatures (around $-55^{\circ} \mathrm{C}$ ) and several cold boot cycles of the system were successfully performed prior to deployment. The string-PCs have been powered-on without problems after extended power-outages at the South Pole.

The AJB allows for over 90 analogue channels to be controlled and read-out without the need to transport the analogue signals over dedicated surface cables to the IceCube Laboratory (ICL). Signal losses and cost are therefore kept to a minimum. Each AJB is connected to the master-PC located in the ICL by two cables (quads) of the IceCube

\footnotetext{
${ }^{6}$ DJ0010G44TK02P10; operating temperatures: $-40{ }^{\circ} \mathrm{C}$ to $+85{ }^{\circ} \mathrm{C}$ (http://www.pqimemory.com/

${ }^{7}$ IDAN-SDM7540HR-8

${ }^{8}$ IDAN-DM6420HR-1-62S

${ }^{9}$ IDAN-DM6952HR-62D
} 


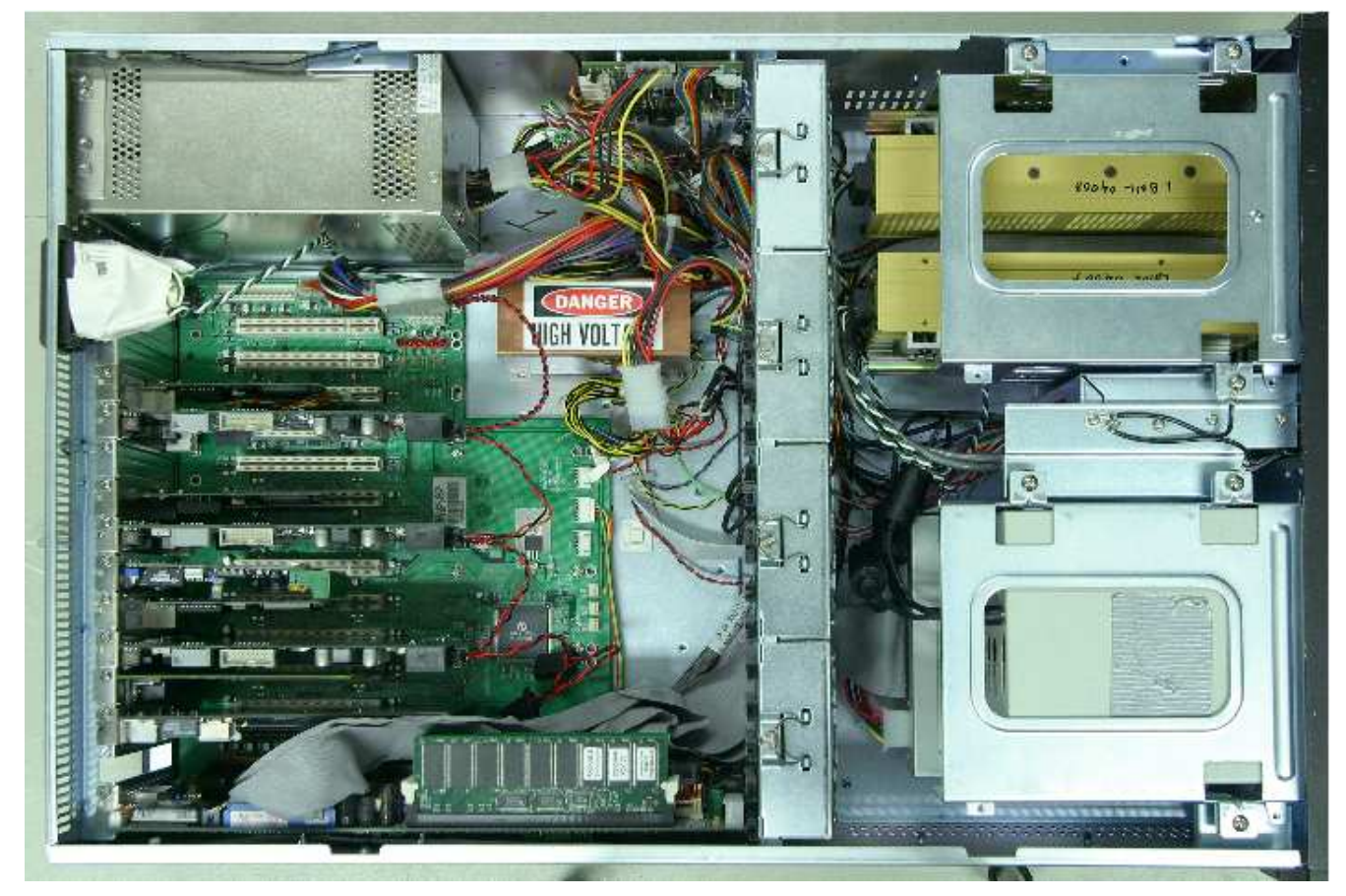

Figure 7: Top view of the master-PC.

surface cable. They each have 2 twisted wire-pairs: DC-power and SDSL 10 communication on one quad and DC-power and a GPS signal on the other. All patch cables are shielded and the shield is connected to the AJB shield. The DC power is automatically inhibited in hardware and firmware if the cable shields are not intact.

\subsection{The master- $P C$}

The master-PC (Fig. 17) is a rackable 4U HP Proliant DL380 series with dual $1 \mathrm{GHz}$ Pentium-3 processors and a 12-slot PCI backplane11. Two Acopian 12 switching regulated power supplies connected in series supply $96 \mathrm{~V}_{\mathrm{DC}}$. They are cooled independently by internal dual fans. A symmetric DSL connection is assured by Nexcomm Nexpeed NM220G modems. These SHDSL modems provide symmetrical data rates of a maximum of $2.3 \mathrm{Mbps}$ for a distance of up to $3 \mathrm{~km}$ over a regular wire-pair. In-situ testing has shown

\footnotetext{
${ }^{10}$ A symmetric DSL connection has the same bandwidth for the upstream and downstream connections.

${ }^{11}$ Retrofitted IceCube Rev 1 DOM Hub.

${ }^{12}$ W48NT370, http://www.acopian.com/
} 
the baseline ping-delay to be around $1.5 \mathrm{~ms}$ for all four strings. A GPS-based IRIG-B (100 pps) time coding signal, provided by a Meinberg GPS PCI card (GPS169PCI) guarantees absolute time stamping. The GPS clock is specified to produce IRIG-B rising edges within $\pm 2 \mu$ s of absolute GPS time. The delay introduced in the IRIG-B signals during propagation from master-PC to string-PC is a few $\mu$ s. Each string has a dedicated PCI control board (SPATS Hub Service Board: SHSB) and ethernet card. The SHSB routes the DSL communication, GPS signal and power to the two surface cable quads. The SHSB firmware can be used to power on and off the strings, as well as to monitor the status of the wire pairs. All minimum and maximum voltages and currents can be set individually and the power is automatically cut-off the instant one of the thresholds is crossed. The master-PC is accessible through the local ICL network. Remote access to that network is only possible when the satellite link with the South Pole Amundson-Scott station is active. Currently, all communication and data-transfer for the entire station are executed over satellites 13 with a total of eight hours of visibility. All SPATS-data is immediately transferred from the string-PCs to the master-PC where it is compressed and stored until transferred. A specialized shell script then prepares different data streams for both satellite transfer and tape archiving. Data transfer capabilities are very limited at the South Pole. SPATS has been assigned a maximum of $150 \mathrm{MB}$ of satellite-transferred data per day. It can take up to five days for the data to arrive on the IceCube data-servers after the data is picked-up by the satellite system. All data that is not transferred automatically goes to tapes that are brought back from South Pole every year.

In addition to the SPATS system at the South Pole, two Northern Hemisphere testsystems also exist: String Z and String E. String Z consists of a complete string-PC that is connected to a desktop-PC (simulating the master-PC) through an SDSL connection. It is possible to connect SPATS sensors, transmitters or a function generator. String E consists of a CPU-board and an fADC board to which a function generator can be attached directly. Both test-systems have been extensively used for DAQ software development.

\footnotetext{
${ }^{13}$ For satellite times, see for example http://ice.rsmas.miami.edu/access.phtml
} 


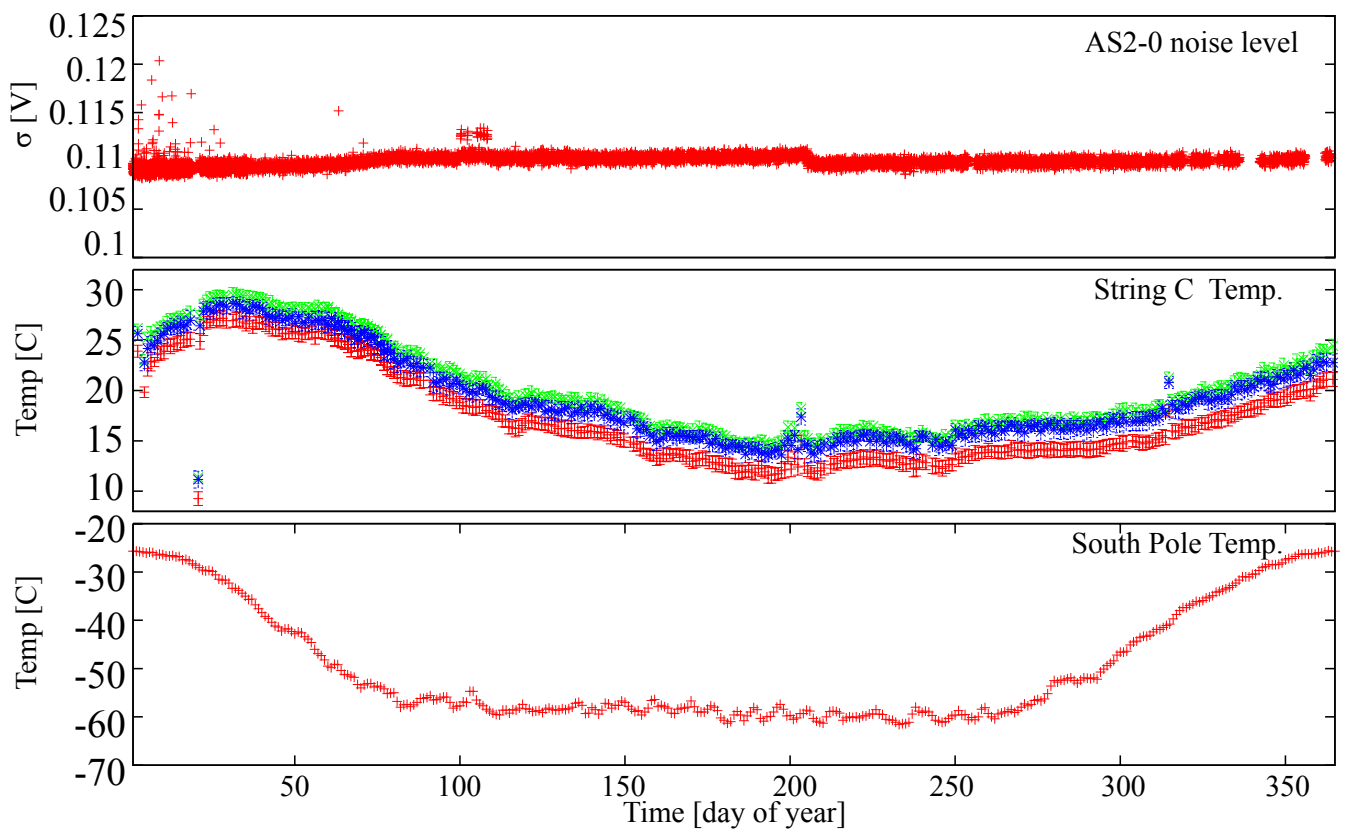

Figure 8: Evolution of the fADC temperatures and AS2-0 noise levels compared to the average surface temperature at the South Pole. The AS2-0 sensor channel is located at a depth of $100 \mathrm{~m}$, at the interface between the firn and the bulk ice.

\section{System performance}

\section{1. $f A D C$ performance}

The temperature of each fADC board is influenced by both external (weather, stationwide power outages) and internal (data-taking modes) factors. The fADC temperatures (and therefore the AJB temperature) take about 12 hours to stabilise after an extended power outage. Over the course of an entire year, the fADC temperature changes by a maximum of $20^{\circ} \mathrm{C}$ whereas the South Pole surface temperatures fluctuates by more than $35^{\circ} \mathrm{C}$. The insulating snow that surrounds the AJB acts as a buffer to the extreme temperatures: the temperature fluctuations are smaller and have a delay of about 1 month, see Fig. 8 ,

Each fADC has a maximum sampling frequency of $1.25 \mathrm{MHz}$. The fADC clocks were found to drift at a rate that is typically several parts per million, this means that the fADC will not sample the waveform at the requested (nominal) frequency but a slightly higher or lower one. The cumulative amount of drift causes decoherence by averaging of 
SPATS transmitter and pinger pulses if the nominal rather than true sampling frequency is used. This clock drift effect can be accounted for by using the IRIG-B GPS signal since the latter is recorded simultaneously with each sensor channel recording. It is therefore possible to determine the actual sampling frequency at the time of the recording. This is the sampling frequency used for subsequent analyses.

\subsection{Current system status}

The status of the sensor channels can easily be assessed by investigation of the ADCcount histogram for noise. Currently 6 out of a total of 80 sensor channels are continuously saturated (AS3 and CS1) and disregarded for analysis. The status of the transmitters can be assessed by looking at their HVRB. Most of the transmitters from strings A, B and C have evolved after their deployment in the ice from displaying smooth HVRB-pulses to more structured ones. The pulses remain very reproducible although the amplitudes typically have decreased. Currently, all but one of the SPATS transmitters are operational.

\section{Data Acquisition}

\subsection{Data format}

All strings run exactly the same software at any given time. An electronic logbook keeps track of all activity on the master and string-PCs.

All waveform data is recorded in a binary format and is automatically assigned an incremental run-number. A SPATS run consists of a certain number of events that can each contain a sensor waveform and/or transmitter HVRB waveform. A waveform consists of samples that contain both the IRIG-B and ADC counts so that each sample has precise time stamping.

\subsubsection{System monitoring data-taking}

The monitoring data allows the basic system parameters to be controlled for both the master-PC and the string-PCs. The string-PC monitoring script runs once per hour and collects the following data: 
1. PTS monitoring: the pressure and temperature sensors inside the transmitter modules are read,

2. fADC temperatures: the internal temperature sensor is read-out for all fADC boards,

3. Network Time Protocol (NTP) variables. Jitter on the system time NTP synchronisation is monitored. The jitter is a measure of the phase noise in the time received from the server, if the time queries happen at uneven intervals, the jitter is high,

4. The string-PC DOM and RAM-disk status.

The daily runlog keeps track of the start- and stop-times of all runs. The master-PC monitoring script runs each hour and collects the following data:

1. power monitoring: the status of the wire pairs for all SPATS surface cables is monitored,

2. ping-delays: the average ping time between the string-PCs and the master-PC is recorded,

3. the master-PC hard disk status,

4. the status of the NTP sever on the master-PC.

The results of the system monitoring data-taking are compiled in an email (monitormail) and sent over the Iridium cellular satellite network during satellite downtimes so that action can be taken right from the start of the next satellite pass. In addition, all data-transfer details are compiled in another email. Herein, the total data-transfer rate for that day and the amount of data that went to the local taping system, without being transferred to the north, are given.

\subsubsection{Acoustic data-taking}

There are two main C-programs that can be used for a variety of acoustic datataking modes. In most modes, the three channels of each sensor module are recorded simultaneously and the data-taking loops over all stages of the string. This scheme allows each sensor channel to be recorded at a sampling frequency of $200 \mathrm{kHz}$. The data taking modes are: 
- Untriggered noise. The noise waveform is recorded for a short duration (currently $0.1 \mathrm{~s}$ ) at a sampling frequency of $200 \mathrm{kHz}$. These full waveforms are used for the absolute noise level analysis, since they retain all frequency information up to $100 \mathrm{kHz}$.

- Triggered noise. If the number of ADC counts on any of the twelve monitored channels (three on each string: one for each ADC board) exceeds a certain level above noise, we record a $5 \mathrm{~ms}$ window of data (1001 ADC samples at $200 \mathrm{kHz}$ ) centered on the trigger in that channel. The resulting trigger rate is roughly stable and of the order of a few triggers every minute for each of the monitored channels. Most of these events are Gaussian noise events, where only one sample is outside the trigger boundaries. The transient events are processed off-line and analysed for time-coincidence clustering. This also allows monitoring of the fADC clock drifts and the recording of noise-histograms during transient data-taking.

- Untriggered noise histograms. It was found that it was desirable to have a way of monitoring the noise level for all sensor channels without having to save the entire waveform for each channel. Indeed, this last option uses a large amount of disk space and SDSL transfer time. Therefore, the noise is additionally recorded in the form of an ADC-count histogram.

- Intra-stage, intra-string and inter-string. A sensor records the acoustic pulses originating from the transmitter on the same stage, same string or on a different string. In a typical inter-string data-taking schedule, one transmitter is triggered while all of the other strings loop over their sensors. This way, all transmitter-sensor combinations are obtained.

- Pinger runs. A sensor records the acoustic pulse originating from the retrievable transmitter. For the 2008/2009 pinger season, the pinger signal is recorded for $18 \mathrm{~s}$ at a sampling frequency of $200 \mathrm{kHz}$ by all three channels of the sensor module simultaneously. A string completes a loop over all sensor modules in less than 4 min. The 4 SPATS strings can record the same module at the same time within $10 \mathrm{~ms}$ due to the NTP synchronisation and the fact that the data-taking script is restarted every $4 \mathrm{~min}$. 
Since the observed noise in the SPATS sensors is Gaussian, the noise levels in the ice can be monitored by looking at the evolution of the standard deviation of the Gaussian ADC-count distributions for each channel. The SPATS noise levels are found to be very stable. One exception to this is that noise levels were observed to increase during the IceCube drilling season. In fact, it was shown that the drill can clearly be heard by the SPATS sensors if it is close enough. Fig.9 shows the width of the ADC-count distributions for all available sensor channels of String B for a period during which the IceCube drill was passing by at the depths of each of the stages.

Currently the SPATS data-taking is concentrating on transient data-taking: 45 minutes of each hour are dedicated to transient data-taking, the remaining $15 \mathrm{~min}$ are reserved for noise data-taking and system monitoring. The thresholds for each channel that takes part in transient data-taking are chosen so that the total data-rate for SPATS remains under $150 \mathrm{MB} /$ day under normal trigger circumstances. In total, over $700 \mathrm{~GB}$ of data has been accumulated over the past $\sim 4$ years.

\section{Calibration and tests}

Prior to deployment the sensitivity in water and equivalent self-noise level of all sensors of strings A, B, and C were measured. At the South Pole the sensors are exposed to low temperatures $(-50 \mathrm{C})$, increased static pressure, and a different acoustic coupling from the medium to the sensor. The change of sensor sensitivity with static pressure and temperature has been studied in dedicated laboratory experiments. We are currently investigating how the sensitivity, as determined in water, can be transferred to the insitu conditions in the Antarctic ice.

A present estimate for the average SPATS sensor sensitivity in ice uses information from laboratory studies of temperature and pressure dependence on this quantity (see section 7.4) as well as data from the noise behaviour during sensor freeze-in at the South Pole. The derived value has however a big uncertainty. With a few sensor channels calibrations have been performed in a laboratory ice tank [13]. The data show that the frequency dependence of the sensitivity changes due to damping of mechanical resonances. Results of forthcoming new measurements of this type will hopefully allow to reduce the error on the sensor sensitivity determination in ice considerably. 


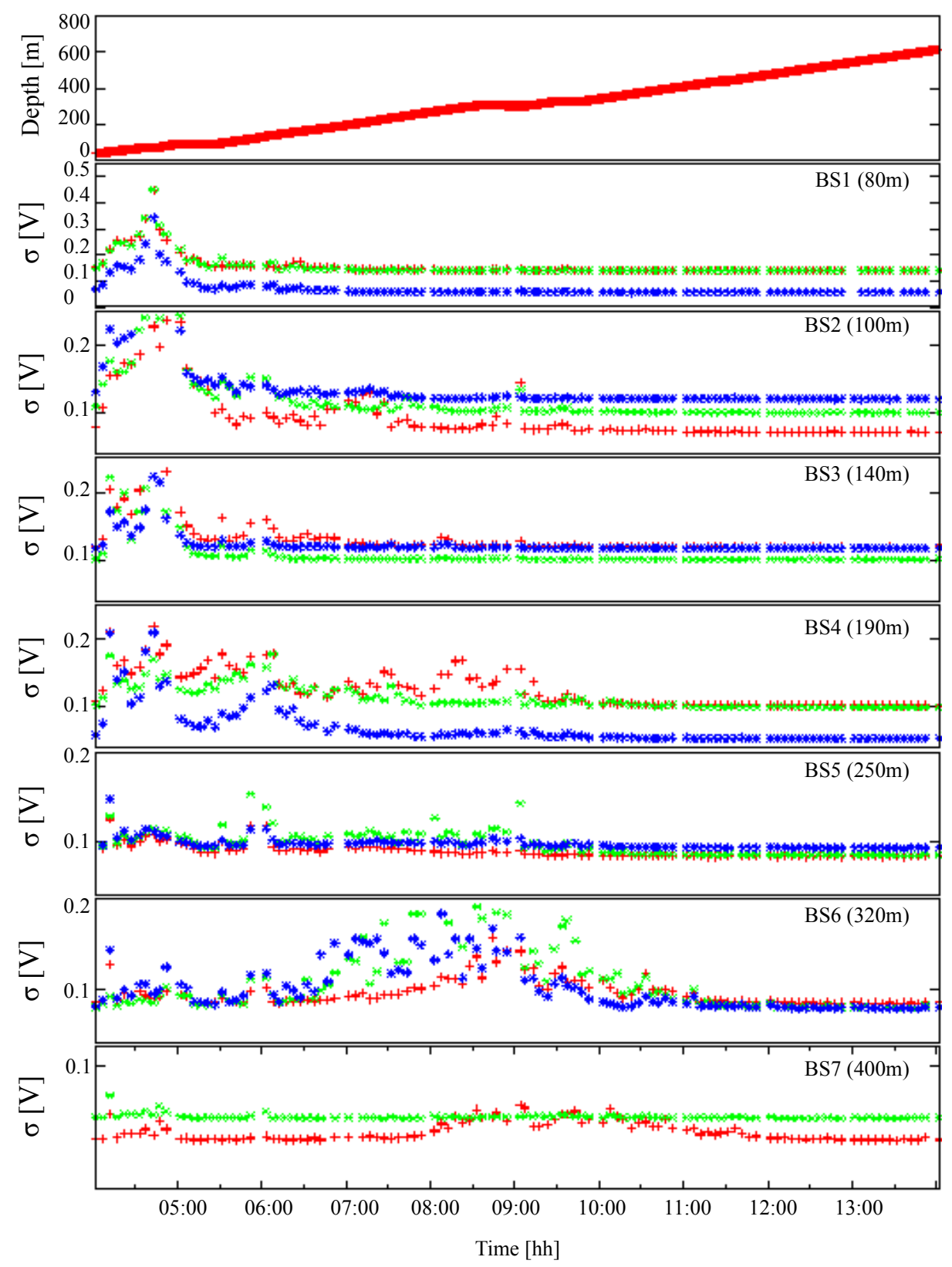

Figure 9: Evolution of the SPATS noise levels for String B during drilling at IceCube hole 64, at a distance of roughly $128 \mathrm{~m}$. The upper plot shows the depth of the IceCube hot-water drill versus time. The width of the Gaussian fitted to the histogram of the ADC voltages is shown for all available sensor channels. 


\subsection{SPATS sensor calibration in water}

The SPATS sensors are calibrated relative to a commercial hydrophon 14 with known spectral sensitivity. This reference hydrophone has a rather flat sensitivity curve for frequencies from $10 \mathrm{kHz}$ to $80 \mathrm{kHz}$ with a mean sensitivity of $(-167.5 \pm 0.3) \mathrm{dB}$ re. $1 \mathrm{~V} / \mu \mathrm{Pa}$.

Ring shaped piezo ceramic elements, similar to the SPATS transmitters, were used to generate a broadband pulse in a water tank at the Hamburgische Schiffbau-Versuchsanstalt (HSVA, Hamburg Ship Model Basin). The available water volume of $12 \times 10 \times 5 \mathrm{~m}^{3}$ is sufficiently large to allow clear separation between direct signals and wall reflections. The acoustic devices were mounted at a depth of $2 \mathrm{~m}$ and the spacing between the transmitter and the sensor was $1.03 \mathrm{~m}$, in order to avoid near field effects. The water temperature was $0.5^{\circ} \mathrm{C}$ and the salinity was $7 \mathrm{ppt}$. Acoustic pulses were recorded with the reference hydrophone and the frequency spectrum of the pressure wave was derived. The SPATS sensor modules were positioned in the same acoustic field. The sensor channel under investigation always pointed towards the transmitter.

In order to cancel the expected contributions of background noise, 100 pulses were recorded for all channels. The uncertainty on the resulting signal frequency spectrum was calculated by dividing each recorded signal into one signal region and four off-signal regions. A discrete Fourier transform of the received signals results in fixed amplitudes $A_{i}$ and phases $\phi_{i}$ for the Fourier coefficients, which are Gaussian smeared by noise contributions (see Fig. (10). The width of the two-dimensional Gaussian $\sigma_{\text {signal }}$ consists of noise contributions $\sigma_{\text {noise }}$ and variations of the pressure pulse shape $\sigma_{\text {pulse }}$. From the difference of $\sigma_{\text {noise }}$ and $\sigma_{\text {signal }}$ it was clear that there was no significant contribution from pressure pulse variation (see Fig. 11). The signal amplitudes were derived from the Fourier coefficient distributions and the sensitivity spectra for all channels were obtained as the ratio of the amplitude spectra and the previously determined pressure wave frequency spectrum. The range of measured sensitivities for all SPATS sensors is shown in Fig. 12 , The mean sensitivity averaged over all sensors and frequencies is $2.6 \cdot 10^{-6} \mathrm{~V} / \mu \mathrm{Pa}$ equal to $-112 \mathrm{~dB}$ re. $1 \mathrm{~V} / \mu \mathrm{Pa}$.

\footnotetext{
${ }^{14}$ SensorTech SQ03
} 


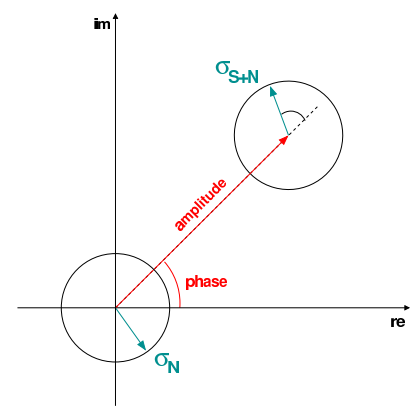

Figure 10: Schematic of noise contributions in the complex plane with signal amplitudes $A$ and phase $\phi$

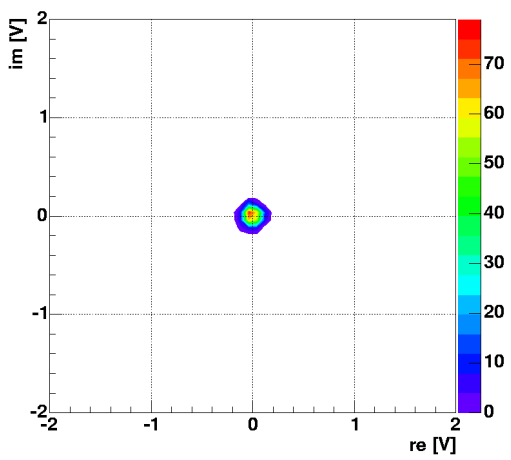

(a)

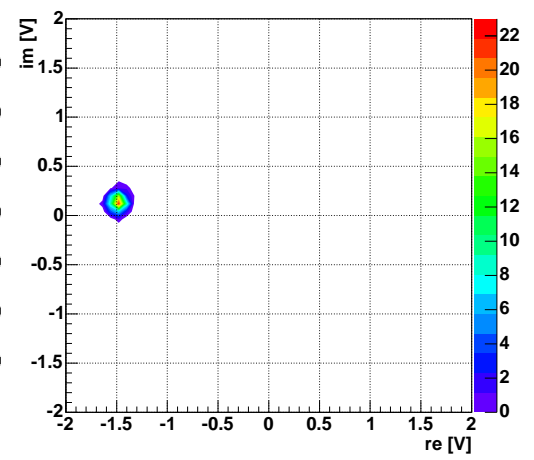

(b)

Figure 11: Distribution of Fourier coefficients for a single sensor channel and one exemplary frequency. Off-signal (noise) contributions (a) and signal contributions (b) are shown. No significant contribution from pressure pulse variations is observed.

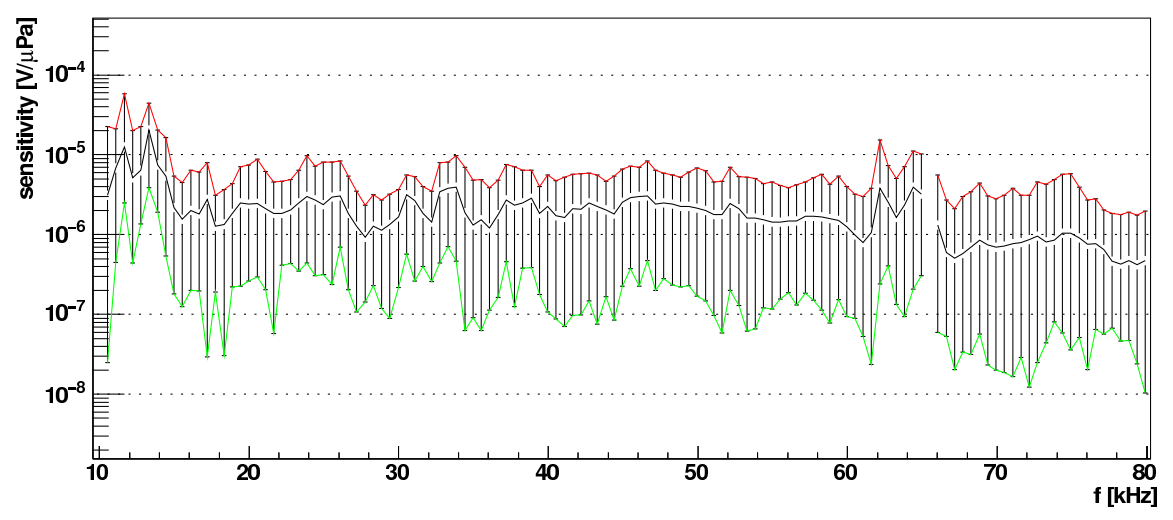

Figure 12: Compilation of all sensor channel sensitivities. The upper and lower lines indicate the highest and lowest sensitivities respectively; the black middle line corresponds to the mean value. 


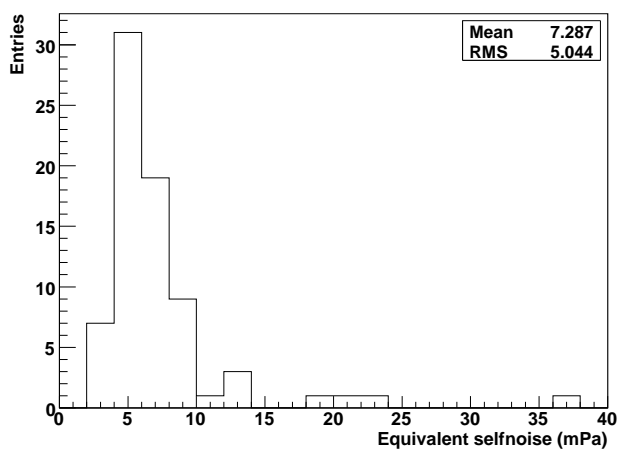

Figure 13: Compilation of equivalent self-noise levels integrated from $10 \mathrm{kHz}$ to $50 \mathrm{kHz}$ for all SPATS channels.

\subsection{Sensor equivalent self-noise}

For each SPATS sensor channel self-noise measurements were performed. External acoustic and electromagnetic interferences was minimized by appropriate shielding. Using the sensor sensitivity, the self-noise frequency spectrum was transformed to an equivalent self-noise spectrum. Integration of this equivalent self-noise spectrum over the frequency range of interest resulted in the equivalent self-noise level. This was used as a measure for the threshold above which acoustic waves were detectable by the sensor. In Fig. 13 the equivalent self-noise level integrated from $10 \mathrm{kHz}$ to $50 \mathrm{kHz}$, the frequency range of interest for acoustic neutrino detection, for all SPATS channels is shown.

\subsection{Transmitter directivity calibration}

Azimuthal isotropic emission is the motivation for the use of ring shaped piezo ceramics. The actual emission directivity of a ring shaped piezo ceramic inside epoxy was measured in azimuthal and polar direction. For measuring polar variations, the piezo ceramic was turned around an axis perpendicular to the ring symmetry axis. The pulses were recorded by the reference hydrophone at a distance of $1 \mathrm{~m}$. Figure 14 shows the received pulse amplitudes as function of azimuth and polar angle. Figure 14](c) shows a schematic for the azimuthal directivity measurement. It illustrates how the positioning of the piezo inside the epoxy could tilt the piezo away form the azimuthal plane.

During deployment in South Pole ice, there is no control over the azimuthal orientation. Amplitude variations of around $40 \%$ are expected due to this systematic effect. 


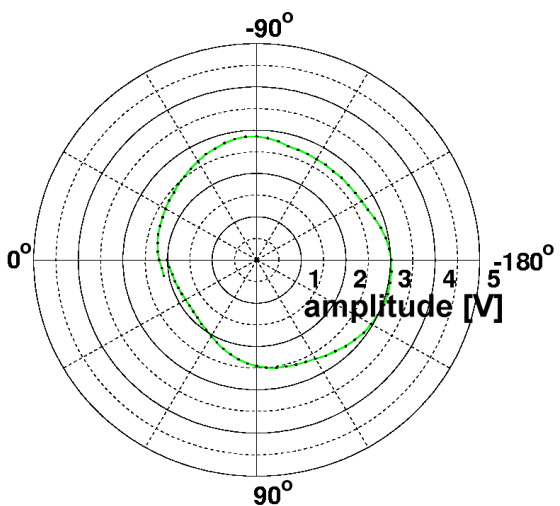

(a)

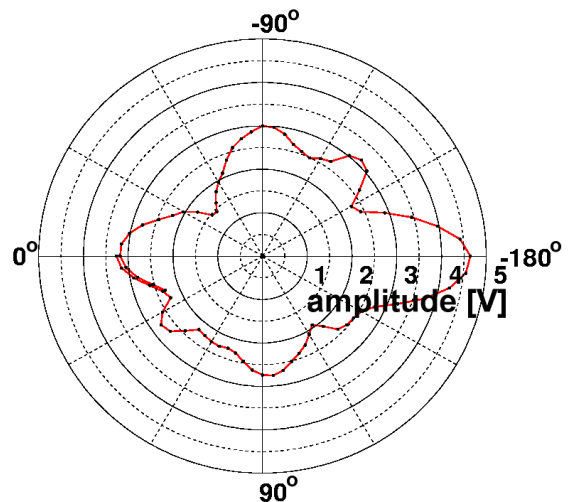

(b)

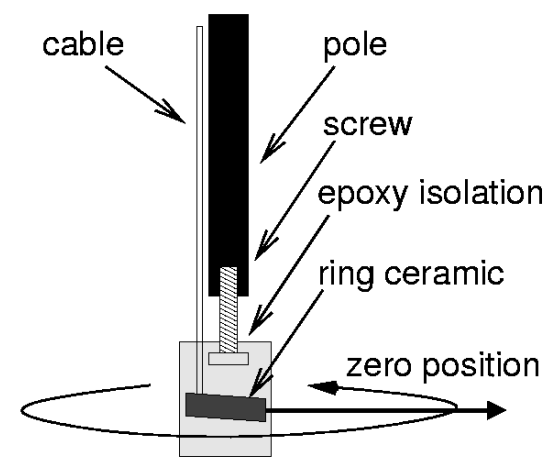

(c)

Figure 14: Received pulse amplitudes as function of transmitter (a) azimuthal and (b) polar orientation. (c) shows the setup for the azimuthal measurement.

The variation of the amplitude outside the horizontal plane in the ice is defined by the polar emission pattern. Despite exhibiting a large variation over the total range, on small scales the polar emissivity is smooth. For repetitive measurements that sample the polar range with a variation of no more than $\pm 10^{\circ}$, the amplitude variation will be below $10 \%$.

\subsection{Pressure and temperature dependence}

\subsubsection{Pressure}

The deepest SPATS stage is deployed at a depth of $500 \mathrm{~m}$ and the maximum ambient pressure is expected to be 100 bars. This is due to the fact that the water inside the 
IceCube hole refreezes first at the surface, creating over-pressure during the freeze-in. The SPATS steel pressure housings have been tested at static pressures up to $120 \mathrm{bar}$, which is roughly the equivalent of $1200 \mathrm{~m}$ of water depth. The housings are not expected to be deformed by more than roughly $50 \mu \mathrm{m}$ at a tension of $180 \mathrm{~N} / \mathrm{m}^{2}$. It is possible that a slight deformation gives rise to a change in sensitivity. For example, the preload on a piezo-ceramic element could change due to deformation of the steel housing.

In order to quantify the change in sensitivity due to the increase of ambient pressure, two pressure test sequences were done at Uppsala University, in Sweden, in August 2008 and February 2009. A large (40.5 cm inner diameter) pressure vessel was used in which SPATS or HADES sensors were consecutively installed. A commercial transmitter (SQ9) was mounted outside the pressure vessel for the February 2009 tests. This data-set consisted of pressure dependent measurements of the transmitter pulse at different operating voltages. The transmitter was pulsed with a single-cycle $20 \mathrm{kHz}$ sine wave. These signals were recorded by the sensors inside the pressure vessel at a maximum pressure of $100 \mathrm{bar}$. The typical step in pressure was 20 bar. The recorded peak-to-peak voltage (Vpp) served as a measure of sensitivity at the various pressure levels. Figure 15(a) shows the measured Vpp for the three channels of a SPATS sensor as a function of ambient pressure: no systematic variation with pressure, common to all three channels, is visible. The complete pressure data set indicated that the variation of sensitivity of a SPATS sensor channel with static pressure was less than $30 \%$ between 1 bar and 100 bar.

\subsubsection{Temperature}

The mean ambient temperature in the South Pole ice in the upper few hundred meters is expected to be around $-50^{\circ} \mathrm{C}$. Several temperature tests have investigated the temperature dependence of the SPATS sensors. To this end, industrial freezers capable of reaching temperatures below $-60^{\circ} \mathrm{C}$ were used. In an experimental verification, performed in 2009, an ITC1001 transmitter was pulsed in air while being suspended near the freezer at the DESY laboratory in Zeuthen, Germany. A SPATS sensor was positioned on a support at the bottom of the freezer. The position of the sensor was not changed between the different measurements. The temperature for each measurement was taken with a thermocouple and a digital thermometer which were both in contact with the steel housing of the sensor. The difference between the two temperature measurements 


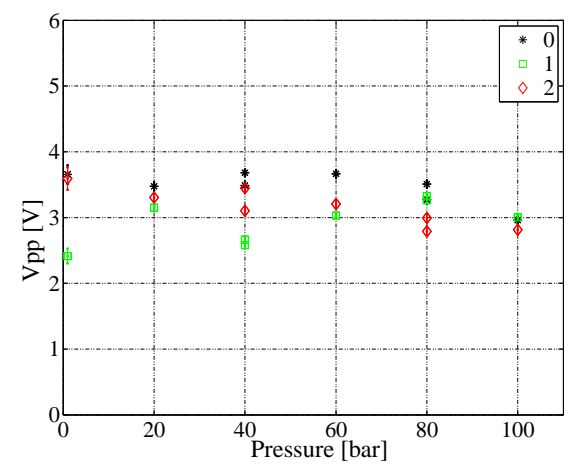

(a)

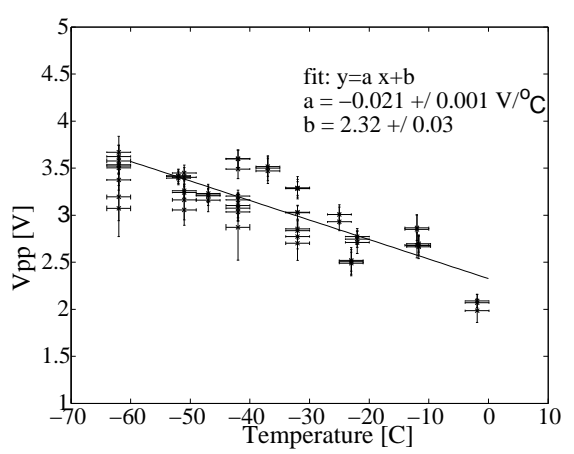

(b)

Figure 15: (a): The peak-to-peak voltage ( $\mathrm{Vpp}$ ) as a function of applied static pressure for the three channels of the same SPATS sensor listening to a transmitter external to the pressure vessel. (b): Vpp as a function of temperature for the three channels of the same SPATS sensor in air with a linear fit to the data (black line).

was typically $2{ }^{\circ} \mathrm{C}$ which was used as the error. The peak-to-peak amplitudes were extracted as the mean of 100 events with the standard deviation as the statistical error. Figure 15(b) shows the extracted Vpp as a function of temperature for the three channels of a same SPATS sensor module. A linear fit was made to the data and it was concluded that there was a gain in the sensitivity of $1.5 \pm 0.2$ when the temperature was lowered from $0{ }^{\circ} \mathrm{C}$ to $-50{ }^{\circ} \mathrm{C}$.

\subsection{Abisko lake test}

A SPATS full system test was performed in lake Torneträsk in the Abisko national park in northern Sweden in April 2006. At the test location the lake was between $40 \mathrm{~m}$ and $60 \mathrm{~m}$ deep and was covered with about $90 \mathrm{~cm}$ of ice. Sensors and transmitters were deployed and read out using a portable DAQ system. Holes were drilled in the ice on a straight line from north to south, where the maximum distance was about $800 \mathrm{~m}$, and two holes were located at $400 \mathrm{~m}$ west and $400 \mathrm{~m}$ east. The goals of this test were threefold: firstly the transmitters were tested to investigate variations between different modules and to demonstrate that the range is sufficient to meet SPATS requirements. Secondly, the performances of the sensor modules were compared to that of the reference hydrophone and the direction of the pulse was reconstructed using two channels 


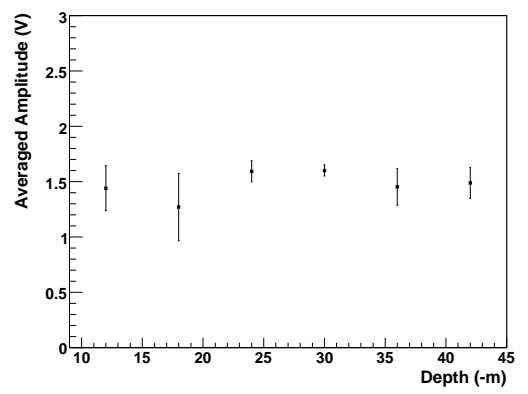

(a)

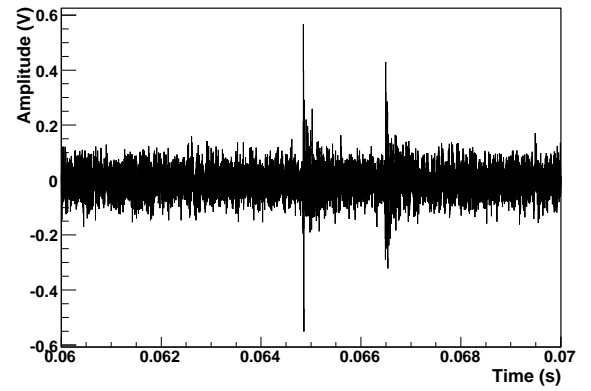

(b)

Figure 16: Results from the Abisko transmitter test: (a) variation in amplitude for one transmitter at different depths, and (b) transmitter signal as seen by a SPATS sensor at $800 \mathrm{~m}$ distance.

per module. Finally, the DAQ software and hardware were tested under real deployment conditions. For each measurement of a transmitter pulse at a certain distance and depth, 10 events were recorded by the reference hydrophone or one of the SPATS sensors. Different effects contributed to shifts in the arrival time of the signal. Underwater currents make the devices swing or torsion in the support rope makes them spin. For analysis, the signals were therefore shifted in time so that the first amplitude maxima coincide, before extracting the mean amplitudes. Lake Torneträsk was found to be a silent testing environment under good weather conditions with a stable and low background noise level of about $120 \mathrm{mV}$ for the SPATS sensors, excluding occasional snow scooters and strong winds.

At first, a SPATS sensor was placed at a depth of $30 \mathrm{~m}$ and different combinations of transmitter modules and ring shaped piezo ceramics were lowered to varying depths in a hole at a distance of $400 \mathrm{~m}$. The amplitudes of the registered pulses were compared; an example can be found in Fig. 16(a) Signals from all tested transmitters are clearly visible at a distance of $400 \mathrm{~m}$. The observed variations in amplitude lie within the expectations and are due to the uncertainties on azimuthal and polar orientation of the transmitter. A maximum distance of $800 \mathrm{~m}$ between transmitter and sensor was then achieved, where the available cable length was the limiting factor for the range test. In Fig $16(\mathrm{~b})$, the recorded signal with a signal to noise ratio of 5 , is shown. The transmitter was positioned at a depth of $20 \mathrm{~m}$. There is a second pulse visible approximately $1 \mathrm{~ms}$ after the signal. A 


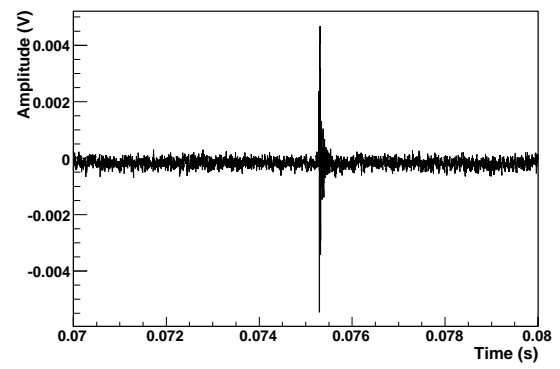

(a)

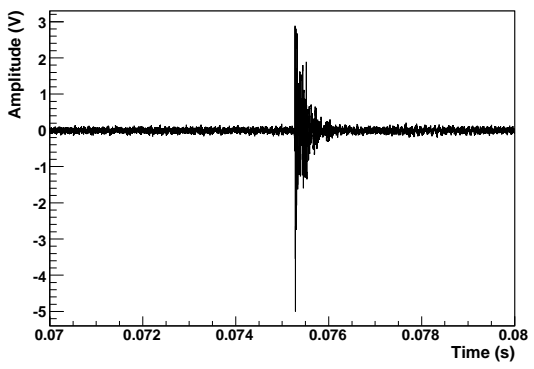

(b)

Figure 17: Sensor results from the Abisko lake test: comparison between the signals received by (a) the commercial hydrophone and (b) SPATS sensor at a distance of $100 \mathrm{~m}$ to the transmitter.

calculation taking into account the speed of sound profile showed that the second pulse is a reflection originating from the ice surface. Assuming an acoustic amplitude attenuation length of $1 \mathrm{~km}$, an extrapolation from the signal to noise ratio gives an expected signal to noise ratio of 1 for the SPATS transmitter in water of $1800 \mathrm{~m}$.

Second, the difference in performance of the SPATS sensor and the reference hydrophone becomes clear in Fig. 17, where the significant difference in scale should be noted. The transmitter was placed at a distance of $100 \mathrm{~m}$ and a depth of $30 \mathrm{~m}$ after which the hydrophone and SPATS sensor were successively lowered to a depth of $16 \mathrm{~m}$. The signal as recorded by the sensor is much stronger than that of the commercial hydrophone. In fact, the hydrophone was incapable of detecting a transmitter signal at a distance $400 \mathrm{~m}$ at the maximum gain setting.

Finally, the SPATS sensor was placed at a depth of $30 \mathrm{~m}$ and a transmitter was lowered to the same depth at positions $400 \mathrm{~m}$ north, west, south and east of the sensor location. The three sensor channels are each separated by $10.5 \mathrm{~cm}$ of steel. The orientation of the sensor module and thus the directional information was obtained by reading out two channels for each position of the transmitter. The difference in arrival times between different channels could be observed at the maximum sampling frequency of $1.25 \mathrm{MHz}$. This way, the sensor channel that was closest to the source could be identified. 


\section{Conclusion and outlook}

The SPATS detector was successfully deployed in the Antarctic ice at the Geographic South Pole and has been recording data for over 5 years. All components of the detector were designed to perform under the extreme temperatures and pressures. No major issues occurred after installation and several unexpected power-outages have not affected system performance. Most of the science goals have been reached and the physics results have both confirmed and challenged theoretical predictions by producing the first ever experimental results for the acoustic attenuation length [12], both the pressure and shear wave sound speeds [11] and the noise levels and transient sounds [14] in the South Pole bulk ice. SPATS is currently undergoing a system upgrade and continues to take acoustic measurements.

\section{Acknowledgments}

We acknowledge the support from the following agencies: U.S. National Science Foundation-Office of Polar Program, Swedish Research Council, Swedish Polar Research Secretariat, and Knut and Alice Wallenberg Foundation, Sweden; German Ministry for Education and Research (BMBF), Deutsche Forschungsgemeinschaft (DFG), Germany; Fund for Scientific Research (FNRS-FWO), Flanders Institute to encourage scientific and technological research in industry (IWT), Belgian Federal Science Policy Office (Belspo); M. Ribordy acknowledges the support of the SNF (Switzerland).

\section{References}

[1] A. Achterberg, et al., First year performance of the IceCube neutrino telescope, Astropart. Phys. 26 (2006) 155-173.

[2] J. A. Aguilar, et al., First results of the instrumentation line for the deep-sea ANTARES neutrino telescope, Astropart. Phys. 26 (2006) 314-324.

[3] I. Kravchenko, et al., Limits on the ultra-high energy electron neutrino flux from the RICE experiment, Astropart. Phys. 20 (2003) 195-213.

[4] L. F. Thompson, J. D. Perkin, Acoustic detection of UHE neutrinos: The ACORNE project, J. Phys. Conf. Ser. 136 (2008) 042070.

[5] P. W. Gorham, et al., Observational Constraints on the Ultra-high Energy Cosmic Neutrino Flux from the Second Flight of the ANITA Experiment, Phys. Rev. D82 (2010) 022004. 
[6] J. Aguilar, et al., Amadeus-the acoustic neutrino detection test system of the antares deep-sea neutrino telescope, Nuclear Instruments and Methods in Physics Research Section A 626-627 (2011) $128-143$.

[7] D. Besson, S. Boeser, R. Nahnhauer, P. B. Price, J. A. Vandenbroucke, Simulation of a hybrid optical/radio/acoustic extension to IceCube for EHE neutrino detection, Int. J. Mod. Phys. A21S1 (2006) 259-264.

[8] P. B. Price, et al., Temperature profile for glacial ice at the South Pole: Implications for life in a nearby subglacial lake., PNAS 99 (2002) 7844-7847.

[9] D. G. Albert, Theoretical modeling of seismic noise propagation in firn at the South Pole, Antarctica, Geophys. Res. Lett. 25(23) (1998) 4257-4260.

[10] B. Semburg for the the IceCube Collaboration , HADES-Hydrophone for Acoustic Detection at South Pole, Nuclear Instruments and Methods in Physics Research A 604 (2009) 215-.

[11] R. Abbasi, et al., Measurement of sound speed vs. depth in South Pole ice for neutrino astronomy, Astropart. Phys. 33 (2010) 277-286.

[12] R. Abbasi, et al., Measurement of Acoustic Attenuation in South Pole Ice, Astropart. Phys. 34 (2011) 382-393.

[13] T. Meures, L. Paul, M. Ribordy for the IceCube Collaboration, Research and Calibration of Acoustic Sensors in Ice Within the SPATS (South Pole Acoustic Test Setup) Project, Nuclear Instruments and Methods in Physics Research A (in press, doi:10.1016/j.nima.2010.10.124).

[14] R. Abbasi, et al., Background studies for acoustic neutrino detection at the South Pole, Submitted to Astropart. Phys. 